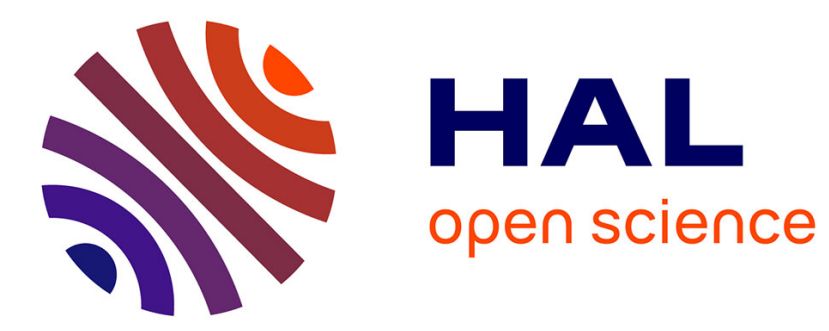

\title{
CAKE: Code-based Algorithm for Key Encapsulation
}

Paulo S. L. M. Barreto, Shay Gueron, Tim Guneysu, Rafael Misoczki, Edoardo Persichetti, Nicolas Sendrier, Jean-Pierre Tillich

\section{To cite this version:}

Paulo S. L. M. Barreto, Shay Gueron, Tim Guneysu, Rafael Misoczki, Edoardo Persichetti, et al.. CAKE: Code-based Algorithm for Key Encapsulation. IMACC 2017 - 16th IMA International Conference on Cryptography and Coding, Dec 2017, Oxford, United Kingdom. pp.207-226, 10.1007/9783-319-71045-7_11. hal-01661949

\section{HAL Id: hal-01661949 \\ https://hal.inria.fr/hal-01661949}

Submitted on 12 Dec 2017

HAL is a multi-disciplinary open access archive for the deposit and dissemination of scientific research documents, whether they are published or not. The documents may come from teaching and research institutions in France or abroad, or from public or private research centers.
L'archive ouverte pluridisciplinaire HAL, est destinée au dépôt et à la diffusion de documents scientifiques de niveau recherche, publiés ou non, émanant des établissements d'enseignement et de recherche français ou étrangers, des laboratoires publics ou privés. 


\title{
CAKE:

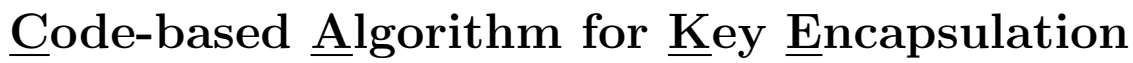

\author{
Paulo S. L. M. Barreto ${ }^{1,2}$, Shay Gueron ${ }^{3,4}$, Tim Güneysu ${ }^{5,6}$, Rafael Misoczki ${ }^{7}$, \\ Edoardo Persichetti ${ }^{8}$, Nicolas Sendrier ${ }^{9}$, Jean-Pierre Tillich $^{9}$ \\ 1 University of Washington Tacoma, USA \\ 2 University of São Paulo, Brazil \\ 3 University of Haifa, Israel \\ 4 Amazon Web Services ${ }^{\star \star}$, USA \\ 5 University of Bremen, Germany \\ 6 DFKI, Germany \\ 7 Intel Corporation, USA \\ 8 Florida Atlantic University, USA \\ ${ }^{9}$ INRIA, France
}

\begin{abstract}
Current widely-used key exchange (KE) mechanisms will be vulnerable to quantum attacks when sufficiently strong quantum computers become available. Therefore, devising quantum-resistant replacements that combine efficiency with solid security guarantees is an important and challenging task. This paper proposes several contributions towards this goal. First, we introduce " $C A K E$ ", a key encapsulation algorithm based on the QC-MDPC McEliece encryption scheme, with two major improvements: a) the use of ephemeral keys that defeats a recent reaction attack against MDPC decoding of the corresponding encryption scheme and b) a highly efficient key generation procedure for QC-MDPCbased cryptosystems. Then, we present an authenticated key exchange protocol based on CAKE, which is suitable for the Internet Key Exchange (IKE) standard. We prove that CAKE is IND-CPA secure, that the protocol is SK-Secure, and suggest practical parameters. Compared to other post-quantum schemes, we believe that CAKE is a promising candidate for post-quantum key exchange standardization.
\end{abstract}

Keywords: post-quantum cryptography, code-based cryptography, key exchange.

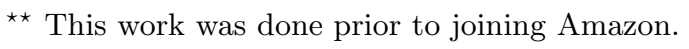

Acknowledgments: Shay Gueron, Tim Güneysu, Nicolas Sendrier and Jean-Pierre Tillich were supported in part by the Commission of the European Communities through the Horizon 2020 program under project number 645622 (PQCRYPTO). Shay Gueron was also partially supported by the Israel Science Foundation (grant No. 1018/16). Paulo S. L. M. Barreto was partially supported by Intel and FAPESP through the project "Efficient Post-Quantum Cryptography for Building Advanced Security Applications" (grant No. 2015/50520-6).
} 


\section{Introduction}

The currently deployed public key cryptosystems rely on the difficulty of number theory problems, e.g. factorization [48] and the discrete logarithm problem [38]. These problems will be efficiently solved by large quantum computers [51, turning those schemes completely useless in a not-so-distant future. Thus, it is of great relevance to devise and deploy alternative schemes that can survive the advent of large quantum computers and, ideally, offer reasonable performance.

In this context code-based cryptography is a promising alternative. It relies on the well-known decoding problem [7], believed to be hard even against quantum adversaries 8 , and on the indistinguishability of its public key from random, a problem that strongly depends on the code family. The best-known code-based scheme, namely the McEliece encryption scheme [36], suggests binary Goppa codes as the code family. This choice has two main drawbacks: a) Goppa codes may not be the optimal security choice given a recent distinguisher for certain Goppa codes [19] and b) they require very large public keys (size of several megabytes).

To address these issues, the QC-MDPC McEliece scheme [40] was introduced replacing Goppa codes by Quasi-Cyclic Moderate-Density Parity-Check (QCMDPC) codes. This approach led to key sizes that are just a few thousand bits long, and approximated the distinguishing problem to the decoding problem. These features attracted great attention from the community (see $26,53,54$, 16, just to mention a few) including a mention in the preliminary European recommendations for post-quantum cryptography 31.

Despite their promising features, QC-MDPC codes need to be handled carefully due to the probabilistic nature of MDPC decoding (which is inherited from Low-Density Parity-Check (LDPC) codes 22]): there is some probability that the MDPC decoding may fail. This property can be leveraged to mount an attack on some schemes. Indeed, Guo, Johansson and Stankovski 23 presented an interesting reaction attack (GJS attack) against the QC-MDPC McEliece encryption scheme. In this attack, the adversary carefully crafts error patterns and observes whether (or not) the decoding process fails. The adversary can recover the private key by collecting the decoding failure rate of various error patterns. This is possible only because the parameters suggested in [40] ensured (through exhaustive simulation) a decoding failure rate (DFR) of $10^{-7}$. The simplest way

to foil this attack is to choose parameters such that DFR $\leq 2^{-\lambda}$, where $\lambda$ is the security level of the scheme. However, the difficulty with this strategy is to formally prove that a given parameter set attains a given DFR. Empirical observations indicate that the DFR of MDPC codes decreases exponentially, and secure parameters could be achieved by increasing the code length of 40 by $30 \%$ or $40 \%$. However, with no formal proof for this property, the GJS attack might prevent wide adoption of QC-MDPC McEliece for asymmetric encryption.

Contributions. Thus far neither an MDPC-based key exchange nor any MDPC-based encryption scheme has been proposed that defeats the GJS attack. This work provides several contributions to address this and other problems: 
$\checkmark$ It introduces CAKE, a new key encapsulation mechanism (KEM), based on QC-MDPC codes. It differs from the QC-MDPC McEliece encryption scheme in two respects: a) the key generation process is significantly faster at the cost of longer public keys, and b) completely defeats the GJS attack by employing ephemeral keys (i.e., new keys are generated at each key exchange).

$\checkmark$ It proposes an authenticated key exchange protocol based on CAKE that is suitable for the Internet Key Exchange (IKE), similarly to what was previously done done for lattices 42 .

$\checkmark$ It proves that CAKE is CPA secure and the protocol is SK secure 13].

The full version of this paper will include a discussion of implementation aspects, including strategies to deploy our proposal in an isochronous way.

Related Work. Lattice-based cryptography has a long record of academic works 43], including promising key exchange (KE) protocols. The NewHope scheme 1] provides good performance and is based on the Ring-LWE problem 32 (a ring variant of the Learning-With-Errors (LWE) problem [47). It improves over previous work by Bos, Costello, Naherig and Stebilla 11] which is an implementation of Peikert's proposal [42] for TLS. Frodo [10] is a key exchange scheme based on the LWE problem itself at the price of larger parameters and worse performance. Cryptography based on isogenies of supersingular elliptic curves seems to be another promising way to devise KE protocols 27, 17 offering small public keys but not so attractive latency. There are very few code-based key encapsulation mechanism (KEM) schemes. The best known is McBits 9 which builds on the work of [45], and lives in the classical McEliece setting with binary Goppa codes and enormous public keys. Very recently, Ouroboros scheme 29] has been introduced and seems to be a competitive proposal with security proof advantages. There is another class of related works which is not focused on key exchange but that shares some similarities on the techniques to achieve compact McEliece public key sizes, e.g. the seminal work based on quasi-cyclic codes 21], based on quasi-dyadic codes [39, 44, 14] and quasi-cyclic low-density parity-check codes $41,4,5,3,2$.

Organization. This paper is organized as follows. Section 2 presents the preliminary concepts, Section 3 introduces CAKE, a new unauthenticated key encapsulation mechanism (KEM) based on QC-MDPC codes, Section 4 presents an authenticated key exchange protocol based on CAKE, Section 5 proves that CAKE is IND-CPA secure and the corresponding authenticated key exchange protocol is SK secure, Section 6 discusses practical security and suggests parameters. Section 7 presents our conclusions.

\section{Preliminaries}

Definition 1 (Linear codes). The Hamming weight of a vector $x \in \mathbb{F}_{2}^{n}$ is the number wt $(x)$ of its nonzero components. $A$ binary $(n, r)$-linear code $\mathcal{C}$ of length $n$, co-dimension $r$ and dimension $k=(n-r)$ is a $k$-dimensional vector subspace of $\mathbb{F}_{2}^{n}$. It is spanned by the rows of a matrix $G \in \mathbb{F}_{2}^{k \times n}$, called a generator matrix 
of $\mathcal{C}$. Equivalently, it is is the kernel of a matrix $H \in \mathbb{F}_{2}^{r \times n}$, called parity-check matrix, i.e. $\mathcal{C}=\left\{c \mid H c^{T}=0\right\}$. The codeword $c \in \mathcal{C}$ of a vector $m \in \mathbb{F}_{2}^{(n-r)}$ is $c=m G$. The syndrome $s \in \mathbb{F}_{2}^{r}$ of a vector $e \in \mathbb{F}_{2}^{n}$ is $s^{T}=H e^{T}$.

Definition 2 (Quasi-cyclic code). An ( $n, r)$-linear code is quasi-cyclic (QC) if there is some integer $n_{0}$ such that every cyclic shift of a codeword by $n_{0}$ places is again a codeword.

When $n=n_{0} r$, for some integer $r$, it is possible and convenient to have both generator and parity check matrices composed by $r \times r$ circulant blocks. A circulant block is completely described by its first row (or column) and the algebra of $r \times r$ binary circulant matrices is isomorphic to the algebra of polynomials modulo $x^{r}-1$ over $\mathbb{F}_{2}$, enabling efficient computations. For example, a parity-check matrix $H$ of an $\left(n_{0} r, r\right)$-quasi-cyclic code can be represented as:

$$
H=\left[H_{0}|\ldots| H_{n_{0}-1}\right], \text { where: } H_{i}=\left(\begin{array}{ccc}
h_{i, 0} & \ldots & h_{i, r-1} \\
\vdots & \ddots & \vdots \\
h_{i, 1} & \ldots & h_{i, 0}
\end{array}\right) \in \mathbb{F}_{2}^{r \times r}
$$

Definition 3 (QC-MDPC codes). An $\left(n_{0}, r, w\right)-Q C$-MDPC code is a quasicyclic code of length $n=n_{0} r$, co-dimension $r$ admitting a parity-check matrix with constant row weight $w=O(\sqrt{n \log n})$.

\section{CAKE: A QC-MDPC KEM with Fast Key-Generation}

In this section we introduce CAKE - an unauthenticated key encapsulation mechanism based on QC-MDPC codes. The strategy to present our scheme as an unauthenticated KEM follows works such as NewHope [10 and BCNS [11]. In this way, authentication and key exchange features are decoupled, allowing flexibility to select (and eventually replace) the choice for each feature. Section 4 describes one way to add the authentication layer on top of CAKE.

CAKE resembles the QC-MDPC McEliece encryption scheme [40] but also has important differences. While QC-MDPC McEliece intends to use long term keys, CAKE relies on ephemeral keys. This means that a new key pair is generated at each key exchange, thus completely defeating the GJS attack 23] which depends on observing a large number of decoding failures for a same private key. Given the new requirement of generating a key pair at every key exchange, a major challenge consisted of investigating novel strategies to accelerate MDPC key generation. To address this issue, we suggest a simple and elegant solution. In contrast to QC-MDPC McEliece (and any quasi-cyclic McEliece variant), CAKE does not compute the inversion of one of its private cyclic blocks and then multiply it by the whole private matrix. Note that this was done to ensure that one of the blocks in the public matrix is the identity block (and thus no need to be transmitted) and to hide the private code structure. Instead, CAKE hides the private code structure by simply multiplying its sparse private matrix 
by any random, dense cyclic block. This turns CAKE key generation into the most efficient process among key generation, encryption and decryption. It is worth mentioning that the QC-MDPC inversion-based key generation can be up to $21 \mathrm{x}$ slower than encryption 34 . Therefore, removing the inversion operation is an excellent strategy to accelerate key generation.

The drawback of this strategy is the doubled public key size since the public key will not have an identity block anymore. We consider that this is an acceptable cost given the significant speedup. Finally, we make use of a simple variant of McEliece, as presented in [15, 37, which swaps the roles of message and randomness in the encryption process to avoid a costly polynomial inversion.

A key encapsulation mechanism (KEM) is composed by three algorithms: GEN which outputs a public encapsulation key $p k$ and a private decapsulation key $s k$, ENCAPS which takes as input an encapsulation key $p k$ and outputs a ciphertext $c$ and a symmetric key $K$, and DECAPS which takes as input a decapsulation key $s k$ and a cryptogram $c$ and outputs a symmetric key $K$ or a decapsulation failure symbol $\perp$. For more details on KEM definitions, we refer the reader to [18.

For a security level $\lambda$, let $r$ be a prime such that $\left(x^{r}-1\right) /(x-1) \in \mathbb{F}_{2}[x]$ is irreducible, $d_{v}$ be an odd integer and $t$ be an integer such that decoding $t$ errors with a uniformly chosen binary linear error-correcting code of length $n=2 r$ and dimension $r$, as well as recovering a base of column weight $d_{v}$ given an arbitrary base of a QC-MDPC code of the same length and dimension, both have a computational cost in $\Omega(\exp (\lambda))$. See Section 6 for a detailed discussion on parameters selection. The CAKE algorithms definition is presented next. Notation: We denote by $\stackrel{\$}{\leftarrow}$ the process of sampling uniformly at random, by $\mathcal{R}$ the ring $\mathbb{F}_{2}[x] /\left\langle x^{r}-1\right\rangle$ and by $\mathbf{K}:\{0,1\}^{n} \rightarrow\{0,1\}^{\ell_{K}}$ the hash function used by encapsulation and decapsulation, where $\ell_{K}$ is the desired symmetric key length.

\section{Algorithm 1. CAKE.GEN:}

- Input: $\lambda$, the target quantum security level.

- Output: the sparse private key $\left(h_{0}, h_{1}\right)$ and the dense public key $\left(g_{0}, g_{1}\right)$.

0 . Given $\lambda$, set the parameters $r, d_{v}, t$ as described above.

1. Generate $h_{0}, h_{1} \stackrel{\$}{\leftarrow} \mathcal{R}$ both of (odd) weight $\mathrm{wt}\left(h_{0}\right)=\mathrm{wt}\left(h_{1}\right)=d_{v}$.

2. Generate $g \stackrel{\$}{\leftarrow} \mathcal{R}$ of odd weight (so $\operatorname{wt}(g) \approx r / 2$ ).

3. Compute $\left(g_{0}, g_{1}\right) \leftarrow\left(g \cdot h_{1}^{T}, g \cdot h_{0}^{T}\right)$.

Let $H$ and $G$ be the quasi-cyclic matrices built from $(r-1)$ cyclic shifts of $\left(h_{0}, h_{1}\right)$ and $\left(g_{0}, g_{1}\right)$ respectively. It is easy to see that $G \cdot H^{T}=0$ and therefore they satisfy the condition to be a generator and a parity-check matrix of the given code: $G \cdot H^{T}=\left[g \cdot h_{1}^{T} \mid g \cdot h_{0}^{T}\right] \cdot\left[h_{0} \mid h_{1}\right]^{T}=g \cdot h_{1}^{T} \cdot h_{0}^{T}+g \cdot h_{0}^{T} \cdot h_{1}^{T}=$ $g \cdot\left(h_{1}^{T} \cdot h_{0}^{T}+h_{0}^{T} \cdot h_{1}^{T}\right)=2 \cdot g \cdot h_{0}^{T} \cdot h_{1}^{T}=0$. It is also important to show that $g$, as created above, is always invertible (thus not risking to generate a public code which is in fact a sub-code of the private one) and this is proven in Appendix A. 


\section{Algorithm 2. CAKE.EnCAPS:}

- Input: the dense public key $\left(g_{0}, g_{1}\right)$.

- Output: the encapsulated key $K$ and the cryptogram $c$.

1. Generate an error pattern $e=\left(e_{0}, e_{1}\right) \stackrel{\$}{\leftarrow} \mathcal{R}$ of total weight $\mathrm{wt}(e)=t$.

2. Generate $m \stackrel{\$}{\leftarrow} \mathcal{R}$.

3. Compute $c=\left(c_{0}, c_{1}\right) \leftarrow\left(m \cdot g_{0}+e_{0}, m \cdot g_{1}+e_{1}\right)$.

4. Compute $K \leftarrow \mathbf{K}(e)$.

\section{Algorithm 3. CAKE.DECAPS:}

- Input: the sparse private key $\left(h_{0}, h_{1}\right)$ and the cryptogram $c$.

- Output: the decapsulated key $K$ or a failure symbol $\perp$.

1. Compute the syndrome $s \leftarrow c_{0} \cdot h_{0}^{T}+c_{1} \cdot h_{1}^{T}$.

2. Try to decode $s$ to recover an error vector $e^{\prime}=\left(e_{0}^{\prime}, e_{1}^{\prime}\right)$.

3 . If $\operatorname{wt}\left(e^{\prime}\right) \neq t$ or decoding fails, output $\perp$ and halt.

4. Compute $K \leftarrow \mathbf{K}\left(e^{\prime}\right)$.

All public key, private key and cryptogram are $n$ bits long. We remark that $e$ can be represented with only $\left\lceil\log _{2}\left(\begin{array}{l}n \\ t\end{array}\right)\right\rceil$ bits and such a compact representation can be used if memory is the preferred metric of optimization (the hash function $\mathbf{K}$ would need to be changed as well to receive $\left\lceil\log _{2}\left(\begin{array}{l}n \\ t\end{array}\right)\right\rceil$ bits instead of $n$ ). Figure 1 illustrates CAKE as a protocol of messages exchanged between an Initiator and a Responder. Table 1 shows the bandwidth cost per message.

\begin{tabular}{|c|c|c|}
\hline \multirow{3}{*}{$\begin{array}{l}\text { Initiator } \\
\left(h_{0}, h_{1}, g_{0}, g_{1}\right) \leftarrow \text { CAKE.GEN }(\lambda)\end{array}$} & & Responder \\
\hline & $\left(g_{0}, g_{1}\right)$ & \\
\hline & $c$ & $(K, c):=$ CAKE.ENCAPS $\left(g_{0}, g_{1}\right)$ \\
\hline$K / \perp:=\operatorname{CAKE} \cdot \operatorname{DECAPS}\left(h_{0}, h_{1}, c\right)$ & & \\
\hline
\end{tabular}

Fig. 1: CAKE Key Encapsulation Mechanism

\begin{tabular}{|c|c|c|}
\hline Message Flow & Message & Size (bits) \\
\hline \hline Initiator $\rightarrow$ Responder & $\left(g_{0}, g_{1}\right)$ & $n$ \\
Responder $\rightarrow$ Initiator & $\left(c_{0}, c_{1}\right)$ & $n$ \\
\hline
\end{tabular}

Table 1: Communication Bandwidth. 


\section{An Authenticated Key Exchange Protocol From CAKE}

In this section, we discuss one way to extend CAKE to an authenticated key exchange protocol. This discussion intends to demonstrate that CAKE ephemeral keys are not a limitation for its integration into real-world key exchange protocols and also allows us to discuss interesting security properties required in the real world, such as perfect forward secrecy, which are usually managed in levels of abstraction above the simple key encapsulation building block.

The construction here described is based on the well-known SIGn-and-MAc (SIGMA) protocol design 30], which is adopted by the Internet Key Exchange (IKE) protocol [25], part of the IPSec standard [50]. The simplest SIGMA protocol is known as $\overline{\Sigma_{0}}$ and is proven to be secure (in terms to be discussed in Section 5.2 when instantiated with Diffie-Hellman key agreement [13]. Our proposal essentially leverages a result presented by Peikert which demonstrated that $\Sigma_{0}$ can be proven secure with any IND-CPA KEM [42], instead of being restricted to Diffie-Hellman.

As in [42, the key exchange protocol here described is parametrized by an (IND-CPA-secure) key encapsulation mechanism KEM with key space $\mathrm{K}$, a digital signature scheme SIG, a pseudorandom function $f: \mathrm{K} \times\{0,1\} \rightarrow \mathrm{K}_{0}$, and a message authentication code MAC with key space $\mathrm{K}_{0}$ and message space $\{0,1\}^{*}$. A successful execution of the protocol outputs a secret key in $\mathrm{K}_{0}$. In our work, we explicitly defines CAKE as the KEM scheme. For the sake of flexibility, we do not specify any particular signature, MAC or pseudorandom function although they all need to meet some minimum security notion (the signature and MAC must be EUF-CMA secure and $f$ must be a secure pseudorandom function; see Section 5). We assume that each party has a long-term signing key for SIG whose corresponding verification key is publicly available and associated to its identity ID. This can be done in terms of certificate authorities and common public key infrastructure.

Key exchange protocols are multiparty protocols activated by messages that are locally processed, leading to new messages being triggered. A session is an invocation of this protocol. Each session is associated to a unique session ID (denoted as sid) and a party can be called the Initiator (with identity $I D_{I}$ ) who first activates the session or the Responder (identity $I D_{R}$ ) who is activated upon receiving a message. For a more detailed discussion on key exchange protocol definitions we refer to 12 . Figure 2 describes how CAKE can be plugged into an authenticated key exchange protocol, similarly as done in 42$]$.

The protocol assumes that Initiator and Responder possess identities $I D_{I}$ and $I D_{R}$, respectively. Initiator generates a unique session identifier sid and a CAKE key pair $\left(s k=\left(h_{0}, h_{1}\right), p k=\left(g_{0}, g_{1}\right)\right)$, and sends $(s i d, p k)$ to Responder, who generates a key $K$ and a ciphertext $C$ using the encapsulation method. The pair $\left(K_{0}, K_{1}\right)$ is generated from $K$ using the pseudorandom function $f$. The tuple $(1, \operatorname{sid}, p k, C)$ is signed using Responder's signing key and a MAC tag is generated from $\left(1\right.$, dif,$\left.I D_{R}\right)$ using key $K_{1}$. The signature, tag, ID $D_{R}$, sid and $C$ is sent to Initiator, who tries to decapsulate $C$. In case of success, Initiator 


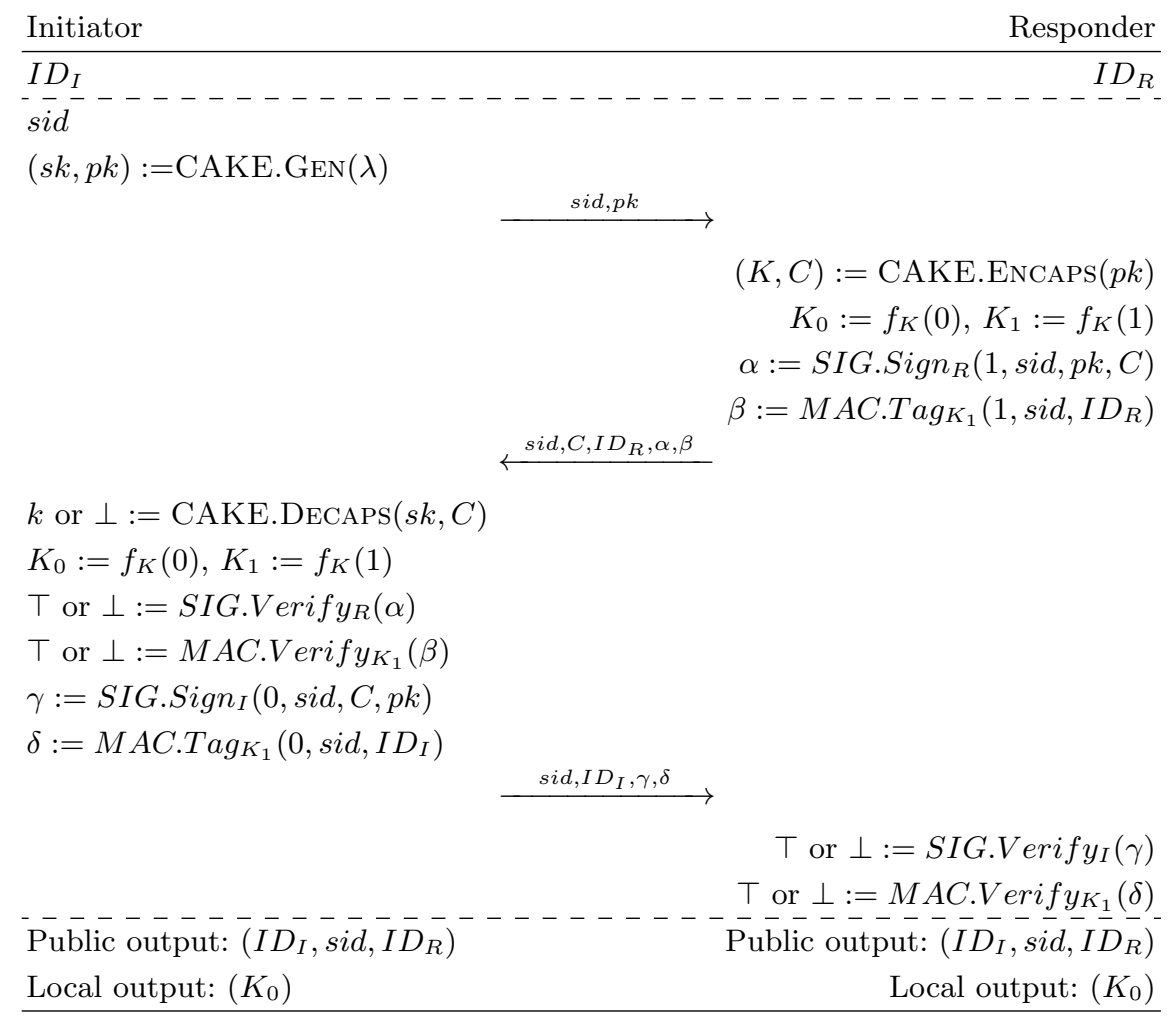

Fig. 2: SIGMA-like Authenticated Key Exchange From CAKE KEM

reconstructs $\left(K_{0}, K_{1}\right)$ and verifies both signature and MAC tag. If it succeeds, Initiator signs the tuple $(0$, sid, $C, p k)$ and generates a MAC tag for the tuple $\left(0\right.$, sid, $\left.I D_{I}\right)$. Signature, tag, sid and $I D_{I}$ are sent to Responder who verifies both signature and tag. If it succeeds, the public output is the tuple $\left(I D_{I}\right.$, sid, $\left.I D_{R}\right)$ and the local output is the shared key $K_{0}$. If any process fails, the public output is (abort, $I D_{I}$, sid) and (abort, $I D_{R}$, sid), and the key exchange is restarted.

\section{Formal Security Assessment}

In this section, we prove that CAKE is IND-CPA secure and that the authenticated key exchange protocol described in Section 4 is SK-Secure.

\subsection{CAKE IND-CPA Security}

In the following definition, we denote by $\mathcal{K}$ the domain of the exchanged symmetric key and by $\lambda$ the security level of the scheme. 
Definition 4. A key encapsulation mechanism is IND-CPA (passively) secure if the outputs of the two following games are computationally indistinguishable.

\begin{tabular}{l|l} 
Game 1 & Game 2 \\
$(s k, p k) \leftarrow \operatorname{Gen}(\lambda)$ & $(s k, p k) \leftarrow \operatorname{GEN}(\lambda)$ \\
$(c, K) \leftarrow \operatorname{EnCAPS}(p k)$ & $(c, K) \leftarrow \operatorname{EnCAPS}(p k)$ \\
& $K^{*} \leftarrow \mathcal{K}$ \\
Output $(p p, p k, c, K)$ & Output $\left(p p, p k, c, K^{*}\right)$
\end{tabular}

Proposition 1. CAKE is IND-CPA secure in the random oracle model assuming that the hash function $\boldsymbol{K}$ is modeled as a random oracle.

Proof. It is easy to show that our claim is true. In fact, note that the only difference between the two distributions in Game 1 and Game 2 is the value of $K$ which is replaced by a uniformly random value $K^{*}$. Remember that in an honest run of CAKE (i.e. Game 1), the key $K$ is obtained as $\mathbf{K}(e)$. Since $\mathbf{K}$ is modeled as a random oracle, its output is pseudorandom. Thus the only way an attacker could distinguish the two values would be by recovering the vector $e$. Since this is a passive attack, the attacker can't perform any decryption queries and can only rely on the public data, that is public key and ciphertext. Thus, this is equivalent to decoding the noisy codeword $c$ in the code described by $\left(g_{0}, g_{1}\right)$. Decoding a random linear code and distinguishing the public code from the private one are both well-known problems which are considered to be hard, and which will be described and discussed extensively in Section 6. For now, suffice to say that since the output of $\mathbf{K}$ is pseudorandom and recovering $e$ is infeasible, CAKE satisfies the IND-CPA security notion.

\subsection{SK Security of Authenticated Key Exchange from CAKE}

The security notion targeted by our SIGMA-like construction and also by 42 is known as SK Secure ${ }^{1}$, which stands for session-key secure 13. Informally, this notion translates into: "the adversary does not learn anything about the session key by interacting with the protocol" and enables the establishment of secure channels (usually the ultimate goal of sharing a key). In the following paragraphs, we give an overview on SK Security.

\footnotetext{
1 This security notion was originally introduced in 12 . The main difference between 12 and 13 is that in the former there was an implicit requirement that the identities of the parties must be known to each other beforehand, while the latter attains a more realistic (internet-oriented) scenario where the identities of the parties are not initially known and only becomes known after the protocol run evolves (this model is called the "post-specified peer model" and is the one used in our proposal).
} 
According to [13, a key exchange protocol is a multiparty protocol where each party runs one or more copies of the protocol. A session is a local procedure resulting from a protocol activation at a party. The activation of a protocol at a party has three inputs $(P, s i d, d)$ : the local party $P$, the unique session identifier sid and the intended peer address $d$. A party can be activated as an Initiator or as a Responder (upon an incoming message). The output of a session is a public triple $(P$, sid, $Q)$, where $Q$ is the intended peer identity and a secret session key. In case of failure, the output is a special failure symbol. Sessions have a local state which is erased after the session completes. Besides, each party may have an additional long-term state (composed by long-term signing keys, for example) which is visible to multiple sessions and is not erased after session completion.

The adversarial model is called the "unauthenticated-links model (UM)" and allows the attacker to have full control over the communication channel, thus being able to intercept, delay, inject, drop, or change any message exchanged. In short, it is a fully-capable man-in-the-middle attacker. Besides, the attacker is also allowed to start key exchange sessions and, more importantly, is able to perform all three session exposure attacks:

- Session-state reveal: targets a still incomplete session. The adversary learns the state of that particular session (not including any long-term secrets accessible to all sessions, such as long-term signing keys).

- Session-key queries: targets a complete session and allows the adversary to learn its corresponding session key.

- Party corruption: the attacker learns all information possessed by the party (including long-term secrets accessible to all sessions, such as longterm signing keys).

An important concept in this model is session expiration. When a session expires, the attacker is not allowed to perform session-state reveal or session-key queries, although is fully able to corrupt a party. A key exchange protocol which is secure even after a party corruption is said to enjoy perfect forward secrecy (PFS). Another relevant concept is the one of matching session.

Definition 5. Let $(P$, sid) be a complete session with public output $(P$, sid, $Q)$. The session $(Q$, sid $)$ is called the matching session of $(P$, sid $)$ if either:

1. $(Q$, sid $)$ is not completed; or

2. $(Q$, sid $)$ is completed and its public output is $(Q$, sid, $P)$.

Finally, the actual concept of SK Secure relies on the attacker's ability of distinguishing a session key from random. This is done through the test session game that allows the attacker to choose any session which has not been exposed (by any of the session exposure attacks above) nor its matching session, and runs the following game used in the formal SK secure definition.

Game 1 (Test Session) Let $\mathcal{U}$ be an adversary of the key exchange protocol $\pi$. In the test-session game, the key exchange protocol oracle toss a coin $b \leftarrow\{0,1\}$ 
and returns a valid session key $k$ if $b=0$ or returns a sequence of random bits if $b=1$. The experiment finishes by the adversary $\mathcal{U}$ outputting $b^{\prime} \in\{0,1\}, a$ guess on the value of $b$.

Definition 6 (SK Secure). A key exchange protocol $\pi$ is SK Secure in the post-specified peer model with unauthenticated links if the following holds for any adversary:

1. $\pi$ satisfies that both uncorrupted parties output the same session key.

2. The probability that $\mathcal{U}$ guesses b correctly in Game 1 is $\frac{1}{2}+\epsilon$, where $\epsilon$ is a negligible fraction in the security parameter.

Having provided this overview on SK Security, we can finally prove the protocol described in Section 4 attains such a security notion.

Theorem 1. The key exchange protocol described in Section 4 is SK Secure in the post-specified peer model with unauthenticated links assuming that:

1. The key exchange protocol described in Section 4 satisfies that both uncorrupted parties output the same session key;

2. CAKE scheme is IND-CPA secure;

3. SIG and MAC are existentially unforgeable under chosen message attack and that the function $f$ is a secure pseudorandom function.

Proof. The proof follows Theorem 6.1 of 42 . The first item is about the correctness of the scheme and boils down to ensure that both parties derive the same session key. This is guaranteed by the correctness of the underlying key encapsulation mechanism (CAKE) and the unforgeability of the signature scheme (see third item below) required to ensure that the key corresponds to the decapsulation of the given ciphertext. As in [42, we remark that the security of the MAC and the pseudorandom function are not needed for such a correctness proof. The second item is achieved by Proposition 5.1. The third item is achieved by construction, i.e. by selecting a MAC and a signature scheme that are EUF-CMA and the function $f$ that is a secure pseudorandom function.

Remark on Perfect Forward Secrecy. Key exchange protocols based on asymmetric encryption, such as key transport protocols, are usually not able to achieve PFS. This happens because if a party is compromised, then its longterm encryption keys are also compromised, allowing the adversary to recover past session keys by decrypting previously exchanged ciphertexts. We remark that this is not the case of the protocol described in Section 4 given the fact we use ephemeral asymmetric encryption keys. Since these keys are part of the session state, they will be erased in an event of session expiration. Signing keys are the actual long-term keys in our proposal and their leakage does not affect previous sessions. The same argument holds for our key encapsulation mechanism (CAKE) as long as the ephemeral encryption keys are guaranteed to be erased after key exchange completion. 


\section{Practical Security Assessment}

This section discusses the practical security aspects of our proposal.

\subsection{Hard Problems and Security Reduction}

Let $\mathcal{R}$ be the ring $\mathbb{F}_{2}[x] /\left\langle x^{r}-1\right\rangle$. For every $h \in \mathcal{R}$ and any positive integer $t$, let $\mathcal{E}(h, t)$ denote the uniform distribution over $\left\{e_{0}+e_{1} h \mid e_{0}, e_{1} \in \mathcal{R}, \operatorname{wt}\left(e_{0}\right)+\right.$ $\left.\mathrm{wt}\left(e_{1}\right)=t\right\}$. For any positive integer $w$, let $\mathcal{K}(w)$ denote the uniform distribution over $\left\{h_{1} h_{0}^{-1} \mid h_{0}, h_{1} \in \mathcal{R}, \operatorname{wt}\left(h_{0}\right)+\operatorname{wt}\left(h_{1}\right)=w\right\}$.

The KEM of $₫ 3$ is secure as long as both distributions $\mathcal{E}(h, t)$ and $\mathcal{K}(w)$ are computationally indistinguishable from the uniform distribution over $\mathcal{R}$. From the practical viewpoint, this means that $r, w, t$ must be chosen such that the following two problems are intractable:

Problem 1. Given $s, h \in \mathcal{R}$, find $e_{0}, e_{1} \in \mathcal{R}$ such that $\operatorname{wt}\left(e_{0}\right)+\operatorname{wt}\left(e_{1}\right)=t$ and $e_{0}+e_{1} h=s$.

Problem 2. Given $h \in \mathcal{R}$, find $h_{0}, h_{1} \in \mathcal{R}$ such that $\operatorname{wt}\left(h_{0}\right)+\operatorname{wt}\left(h_{1}\right)=w$ and $h_{1}+h_{0} h=0$.

Problem 1 and Problem 2 are respectively the problems of decoding $t$ errors and finding a codeword of weight $w$ in an arbitrary quasi-cyclic code of dimension $r$ and length $n=2 r$.

In the current state of the art, the best known techniques for solving those problems are variants of Prange's Information Set Decoding (ISD) 46]. We remark that, though the best attacks consist in solving one of the search problems, the security reduction of our scheme requires the decision version of Problem 2 .

\subsection{Information Set Decoding}

The best asymptotic variant of ISD is due to May and Ozerov [35], but it has a polynomial overhead which is difficult to estimate precisely. In practice, the BJMM variant $[6]$ is probably the best for relevant cryptographic parameters. The work factor for classical (i.e. non quantum) computing of any variant $\mathcal{A}$ of ISD for decoding $t$ errors (or finding a word of weight $t$ ) in a binary code of length $n$ and dimension $k$ can be written

$$
\mathrm{WF}_{\mathcal{A}}(n, k, t)=2^{c t(1+o(1))}
$$

where $c$ depends on the algorithm, on the code rate $R=k / n$ and on the error rate $t / N$. It has been proven in 52 that, asymptotically, for sublinear weight $t=o(n)$ (which is the case here as $w \approx t \approx \sqrt{n}$ ), we have $c=\log _{2} \frac{1}{1-R}$ for all variants of ISD.

In practice, when $t$ is small, using $2^{c t}$ with $c=\log _{2} \frac{1}{1-R}$ gives a remarkably good estimate for the complexity. For instance, non asymptotic estimates derived 
from 24 gives $\mathrm{WF}_{\mathrm{BJMM}}(65542,32771,264)=2^{263.3}$ "column operations" which is rather close to $2^{264}$. This closeness is expected asymptotically, but is circumstantial for fixed parameters. It only holds because various factors compensate, but it holds for most MDPC parameters of interest.

Exploiting the Quasi-Cyclic Structure. Both codeword finding and decoding are a bit easier (by a polynomial factor) when the target code is quasi-cyclic. If there is a word of weight $w$ in a QC code then its $r$ quasi-cyclic shifts are in the code. In practice, this gives a factor $r$ speedup compared to a random code. Similarly, using Decoding One Out of Many (DOOM) [49 it is possible to produce $r$ equivalent instances of the decoding problem. Solving those $r$ instances together saves a factor $\sqrt{r}$ in the workload.

Exploiting Quantum Computations. As commented in [8], Grover's algorithm fully applies to Prange algorithm. Effectively, this halves the above asymptotic exponent for Prange algorithm. Later, it was proven in 28 that more involved variants of ISD could achieve a better exponent but also the improvement was disappointingly away from the factor 2 that could be expected. In the sequel, we will estimate the quantum security by dividing the classical exponent by two. This is probably conservative but a more precise evaluation would not be significantly different.

Practical Parameter Selection. We denote $\operatorname{WF}(n, k, t)$ the workfactor of the best ISD variant for decoding $t$ errors in a binary code of length $n$ and dimension $k$. In the following we will consider only codes of transmission rate 0.5 , that is length $n=2 r$ and dimension $r$. In a classical setting, the best solver for Problem 1 has a cost $\mathrm{WF}(2 r, r, t) / \sqrt{r}$ and the best solver for Problem 2 has a cost $\mathrm{WF}(2 r, r, w) / r$. As remarked above, with $\mathrm{WF}(2 r, r, t) \approx 2^{t}$ we obtain a crude but surprisingly accurate, parameter selection rule. To reach $\lambda$ bits of quantum security, we choose $w, t$ and $r$ such that

$$
\lambda \approx \frac{t-\frac{1}{2} \log _{2} r}{2} \approx \frac{w-\log _{2} r}{2}
$$

\subsection{Defeating the GJS reaction attack}

Both CAKE and the authenticated key exchange protocol described in Section 4 requires ephemeral KEM key pair, i.e. a KEM key generation is performed for each key exchange. As a result, the GJS reaction attack is inherently defeated: a GJS adversary would have (at most) a single opportunity to observe decryption, thus not being able to create statistics about different error patterns. We note that, for efficiency purposes, an initiator may want to precompute KEM key pairs before engaging in key exchange sessions. We remark that policies to securely store the pregenerated KEM key pair must be in place, in order to avoid that an adversary access a KEM key pair to be used in a future communication. 


\subsection{How to Choose MDPC Parameters}

If we denote $\lambda$ the (quantum) security parameter, then both $t$ and $w$ must be close to $2 \lambda$, as in (1). In addition, to ensure decoding, we expect the product $t w$ to grow as $r \log r$. Putting everything together we obtain

$$
\left\{\begin{array}{l}
t \approx 2 \lambda+\log _{2}(2 \lambda) \\
w \approx 2 \lambda+2 \log _{2}(2 \lambda)
\end{array}\right.
$$

and $r$ will grow as $\lambda^{2} / \log \lambda$. The exact value of $r$ needs to be checked, by simulation, and increased to a point where the decoding failure rate is acceptable.

Finally, we choose $r$ such that 2 is primitive modulo $r$. First, this will force $r$ to be prime, thwarting the so-called squaring attack 33. Also, it implies that $x^{r}-1$ only has two irreducible factors (one of them being $x-1$ ). This is an insurance against an adversary trying to exploit the structure of $\mathbb{F}_{2}[x] /\left\langle x^{r}-1\right\rangle$ when $x^{r}-1$ has small factors, other than $x-1$.

The parameters suggested in Table 2 consider the security attacks discussed in Section 6. In addition, the block size $r$ is chosen so that state-of-the-art bit flipping decoding (see [34; or [16 for a comprehensive assessment) has a failure rate not exceeding $10^{-7}$ (validated through exhaustive simulation). The last column shows the public and private key size which are both $n$ bits long.

\begin{tabular}{|c||c|cccc|c|}
\hline$\lambda$ & $n_{0}$ & $n$ & $r$ & $w$ & $t$ & key size (bits) \\
\hline \hline 128 & 2 & 65,542 & 32,771 & 274 & 264 & 65,542 \\
96 & 2 & 39,706 & 19,853 & 206 & 199 & 39,706 \\
64 & 2 & 20,326 & 10,163 & 142 & 134 & 20,326 \\
\hline
\end{tabular}

Table 2: QC-MDPC suggested parameters for $\lambda$ bits of quantum security.

\section{Conclusion}

This paper introduced CAKE, an IND-CPA secure key encapsulation mechanism (KEM) based on QC-MDPC codes. CAKE uses ephemeral keys and therefore inherently defeats the recent GJS attack 23. Since key generation is performed for every key exchange, we devised an efficient QC-MDPC key generation, which is much faster than the one proposed for the QC-MDPC McEliece encryption scheme 40 .

CAKE offers a competitive performance. All public key, private key and cryptogram are $n$ bits long, corresponding to the bandwidth of the messages depicted in Figure 1. The key generation cost is dominated by two sparse-dense modular polynomial multiplications, and does not require any polynomial inversion as usually seen in code-based cryptosystems. The cost of encapsulation is dominated by the two dense-dense modular polynomial multiplications and a hash 
computation. The cost of decapsulation is dominated by two sparse-dense polynomial modular multiplications, a decoding attempt, and a hash computation. In summary, besides MDPC decoding, CAKE relies on modular polynomial multiplications and hash computations, so we can expect efficient implementations on a wide range of platforms. A detailed assessment of implementation aspects will be discussed in the full version of this paper.

CAKE compares well with other post-quantum key exchange schemes. Comparing to other code-based schemes, the Goppa-based McBits 9], one of the fewest currently known code based KEM, offers a public key whose size is orders of magnitude greater than the one of CAKE. From a security perspective, [9] does not seem optimal either given a distinguisher for certain (i.e., high-rate) Goppa codes [19]. On the other hand, Ouroboros [29] is a very recent proposal that seems to have interesting security proof properties.

Recent works [27, 17] have shown that isogenies in supersingular elliptic curves can be used to devise efficient key exchange mechanisms. In particular, those constructions have the benefit of achieving small public key sizes, but not so attractive latency performance. Note that this is a much more recent trend and caution should be exercised as they have not gone through nearly as similar scrutiny as code-based cryptosystems, first appeared almost 40 years ago.

When comparing with lattice-based schemes, e.g., 11, 1] and 10], CAKE and the lattice-based protocols show some similarities. All of them suffer from decoding failures (lattice schemes usually have a lower failure probability though). Also, the use of ephemeral keys for key exchange is not new in the literature; [1] discusses the security loss inherent to key cache (20 presents a comprehensive analysis on the security impact of key reuse for Ring-LWE). Besides, they offer unbalanced cost between the parties, what may lead to great flexibility (e.g., in a certain application, the role of Initiator/Responder could be predefined depending on the expected computational power of the parties). In terms of total bandwidth cost, CAKE's traffic requires $2 / 3$ of the traffic presented in [10], but is 1.3 and 3 times larger than that of [11] and [1], respectively. While such comparisons are certainly useful, we point out that lattice-based schemes are not the immediate "competitors" of CAKE because they are based on a different class of hard problems. We note that the transition to post-quantum cryptography is an unprecedented move, thus, relying on a single, silver-bullet class of cryptographic problems (e.g., lattices) is a very risky strategy, whilst considering a set of well-studied constructions seems a considerably safer choice in the long term.

This paper also presents an SK secure authenticated key exchange protocol based on CAKE, which is suitable for the Internet Key Exchange (IKE), similarly to 42 . We prove that CAKE is IND-CPA secure, and that the authenticated protocol is SK secure. Moreover, we demonstrate that our proposal achieves perfect forward secrecy, despite the fact it is based on asymmetric encryption (key transport schemes with static keys do not attain PFS, for example).

Taking all these considerations into account, we believe that CAKE is a promising candidate for post-quantum key exchange standardization. 


\section{Appendix}

\section{A Efficiently Sampling Invertible Elements from $\mathbb{F}_{2}[x] /\left\langle x^{r}-1\right\rangle$}

In this section, we prove that one can efficiently sample an invertible element from $\mathbb{F}_{2}[x] /\left\langle x^{r}-1\right\rangle$ by taking any polynomial $h \stackrel{\$}{\leftarrow} \mathbb{F}_{2}[x] /\left\langle x^{r}-1\right\rangle$ such that $\mathrm{wt}(h)$ is odd.

Lemma 1. Let $h \in \mathbb{F}_{2}[x]$ have even weight. Then $h$ is not invertible modulo $x^{r}-1$.

Proof. We show that $(x-1) \mid h$ by induction on wt $(h)$. For wt $(h)=0$ trivially $(x-1) \mid h$. Assume that $(x-1) \mid h$ whenever wt $(h)=2 k$ for some $k \geqslant 0$. Now consider any $h \in \mathbb{F}_{2}[x]$ with weight wt $(h)=2(k+1)$, and take two distinct terms $x^{i}, x^{j}$ of $h$ such that $i<j$. Define $h^{\prime}=h-x^{i}-x^{j}$, so that $\operatorname{wt}\left(h^{\prime}\right)=2 k$. Then $(x-1) \mid h^{\prime}$ by induction, i.e. $h^{\prime}=(x-1) h^{\prime \prime}$ for some $h^{\prime \prime} \in \mathbb{F}_{2}[x]$. Hence $h=h^{\prime}+x^{i}+x^{j}=(x-1) h^{\prime \prime}+x^{i}\left(x^{j-i}+1\right)=(x-1) h^{\prime \prime}+x^{i}(x-1)\left(x^{j-i-1}+\cdots+1\right)=$ $(x-1)\left(h^{\prime \prime}+x^{i}\left(x^{j-i-1}+\cdots+1\right)\right)$, and therefore $(x-1) \mid h$.

Theorem 2. Let $r$ a prime such that $\left(x^{r}-1\right) /(x-1) \in \mathbb{F}_{2}[x]$ is irreducible. Then any $h \in \mathbb{F}_{2}[x]$ with $\operatorname{deg}(h)<r$ is invertible modulo $x^{r}-1$ iff $h \neq x^{r-1}+\cdots+1$ and $\operatorname{wt}(h)$ is odd.

Proof. Take a term $x^{i}$ of $h$. Then $\mathrm{wt}\left(h+x^{i}\right)=\mathrm{wt}(h)-1$ is even, and by Lemma 1 $(x-1) \mid\left(h+x^{i}\right)$. Hence $h \bmod (x-1)=x^{i} \bmod (x-1)=1$, meaning that $h$ is invertible modulo $x-1$.

Now, because $\left(x^{r}-1\right) /(x-1)=x^{r-1}+\cdots+1$ is irreducible, if $\operatorname{deg}(h)<r-1$ then $\operatorname{gcd}\left(h, x^{r-1}+\cdots+1\right)=1$, and if $\operatorname{deg}(h)=r-1$, then $\operatorname{gcd}\left(h, x^{r-1}+\cdots+1\right)=$ $\operatorname{gcd}\left(h+x^{r-1}+\cdots+1, x^{r-1}+\cdots+1\right)=1$, since $\operatorname{deg}\left(h+x^{r-1}+\cdots+1\right)<r-1$. Hence $h$ is invertible modulo $x^{r-1}+\cdots+1$.

Therefore, the combination of the inverses of $h$ modulo $x-1$ and modulo $x^{r-1}+\cdots+1$ via the Chinese remainder theorem is well defined, and by construction it is the inverse of $h$ modulo $(x-1)\left(x^{r-1}+\cdots+1\right)=x^{r}-1$.

Corollary 1. One can efficiently sample an invertible element from $\mathbb{F}_{2}[x] /\left\langle x^{r}-\right.$ 1) by taking any polynomial $h \stackrel{\&}{\leftarrow} \mathbb{F}_{2}[x] /\left\langle x^{r}-1\right\rangle$ such that $\mathrm{wt}(h)$ is odd. 


\section{References}

1. Erdem Alkim, Léo Ducas, Thomas Pöppelmann, and Peter Schwabe. Postquantum key exchange - a new hope. Cryptology ePrint Archive, Report 2015/1092, 2015. http://eprint.iacr.org/2015/1092.

2. M. Baldi, M. Bodrato, and F. Chiaraluce. A new analysis of the McEliece cryptosystem based on QC-LDPC codes. In Proceedings of the 6th international conference on Security and Cryptography for Networks (SCN 2008), pages 246-262, Berlin, Heidelberg, 2008. Springer-Verlag.

3. M. Baldi and F. Chiaraluce. Cryptanalysis of a new instance of McEliece cryptosystem based on QC-LDPC codes. In IEEE International Symposium on Information Theory (ISIT 2007), pages 2591-2595, June 2007.

4. M. Baldi, F. Chiaraluce, and R. Garello. On the usage of quasi-cyclic low-density parity-check codes in the McEliece cryptosystem. In Proceedings of the First International Conference on Communication and Electronics (ICEE'06), pages 305-310, October 2006.

5. M. Baldi, F. Chiaraluce, R. Garello, and F. Mininni. Quasi-cyclic low-density parity-check codes in the McEliece cryptosystem. In IEEE International Conference on Communications (ICC 2007), pages 951-956, june 2007.

6. Anja Becker, Antoine Joux, Alexander May, and Alexander Meurer. Decoding random binary linear codes in $2^{n / 20}$ : How $1+1=0$ improves information set decoding. In D. Pointcheval and T. Johansson, editors, Advances in Cryptology EUROCRYPT 2012, volume 7237 of LNCS, pages 520-536. Springer, 2012.

7. E. Berlekamp, R. McEliece, and H. van Tilborg. On the inherent intractability of certain coding problems (corresp.). Information Theory, IEEE Transactions on, 24(3):384 - 386, may 1978 .

8. Daniel J. Bernstein. Grover vs. McEliece, pages 73-80. Springer Berlin Heidelberg, Berlin, Heidelberg, 2010.

9. Daniel J. Bernstein, Tung Chou, and Peter Schwabe. Mcbits: Fast constant-time code-based cryptography. In Lecture Notes in Computer Science (including subseries Lecture Notes in Artificial Intelligence and Lecture Notes in Bioinformatics), volume 8086 LNCS of Lecture Notes in Computer Science (including subseries Lecture Notes in Artificial Intelligence and Lecture Notes in Bioinformatics), pages 250-272, 122013.

10. Joppe Bos, Craig Costello, Léo Ducas, Ilya Mironov, Michael Naehrig, Valeria Nikolaenko, Ananth Raghunathan, and Douglas Stebila. Frodo: Take off the ring! practical, quantum-secure key exchange from LWE. Cryptology ePrint Archive, Report 2016/659, 2016. http://eprint.iacr.org/2016/659

11. Joppe W Bos, Craig Costello, Michael Naehrig, and Douglas Stebila. Post-quantum key exchange for the tls protocol from the ring learning with errors problem. In Security and Privacy (SP), 2015 IEEE Symposium on, pages 553-570. IEEE, 2015.

12. Ran Canetti and Hugo Krawczyk. Analysis of key-exchange protocols and their use for building secure channels. In International Conference on the Theory and Applications of Cryptographic Techniques, pages 453-474. Springer, 2001.

13. Ran Canetti and Hugo Krawczyk. Security analysis of ike's signature-based keyexchange protocol. In Annual International Cryptology Conference, pages 143-161. Springer, 2002.

14. Pierre-Louis Cayrel, Gerhard Hoffmann, and Edoardo Persichetti. Efficient implementation of a cca2-secure variant of McEliece using generalized Srivastava codes. In Proceedings of PKC 2012, LNCS 7293, Springer-Verlag, pages 138-155, 2012. 
15. Pierre-Louis Cayrel, Gerhard Hoffmann, and Edoardo Persichetti. Efficient implementation of a cca2-secure variant of McEliece using generalized Srivastava codes. In Proceedings of PKC 2012, LNCS 7293, Springer-Verlag, pages 138-155, 2012.

16. Julia Chaulet and Nicolas Sendrier. Worst case QC-MDPC decoder for McEliece cryptosystem. In Information Theory (ISIT), 2016 IEEE International Symposium on, pages 1366-1370. IEEE, 2016.

17. Craig Costello, Patrick Longa, and Michael Naehrig. Efficient Algorithms for Supersingular Isogeny Diffie-Hellman, pages 572-601. Springer Berlin Heidelberg, Berlin, Heidelberg, 2016.

18. R. Cramer and V. Shoup. Design and analysis of practical public-key encryption schemes secure against adaptive chosen ciphertext attack. SIAM J. Comput., 33(1):167-226, January 2004.

19. Jean-Charles Faugere, Valérie Gauthier-Umana, Ayoub Otmani, Ludovic Perret, and Jean-Pierre Tillich. A distinguisher for high-rate McEliece cryptosystems. IEEE Transactions on Information Theory, 59(10):6830-6844, 2013.

20. Scott Fluhrer. Cryptanalysis of ring-lwe based key exchange with key share reuse. Cryptology ePrint Archive, Report 2016/085, 2016. http://eprint.iacr.org/ 2016/085.

21. P. Gaborit. Shorter keys for code based cryptography. In International Workshop on Coding and Cryptography (WCC 2005), pages 81-91, Bergen, Norway, 2005. ACM Press.

22. R. G. Gallager. Low-Density Parity-Check Codes. PhD thesis, M.I.T., 1963.

23. Qian Guo, Thomas Johansson, and Paul Stankovski. A Key Recovery Attack on $M D P C$ with CCA Security Using Decoding Errors, pages 789-815. Springer Berlin Heidelberg, Berlin, Heidelberg, 2016.

24. Yann Hamdaoui and Nicolas Sendrier. A non asymptotic analysis of information set decoding. Cryptology ePrint Archive, Report 2013/162, 2013. http://eprint. iacr.org/2013/162

25. Dan Harkins and Dave Carrel. Rfc 2409: The internet key exchange (ike). Status: Proposed Standard, 1998.

26. Stefan Heyse, Ingo Von Maurich, and Tim Güneysu. Smaller keys for code-based cryptography: QC-MDPC McEliece implementations on embedded devices. In International Workshop on Cryptographic Hardware and Embedded Systems, pages 273-292. Springer, 2013.

27. David Jao and Luca De Feo. Towards quantum-resistant cryptosystems from supersingular elliptic curve isogenies. In International Workshop on Post-Quantum Cryptography, pages 19-34. Springer, 2011.

28. Ghazal Kachigar and Jean-Pierre Tillich. Quantum information set decoding algorithms. In Tanja Lange and Tsuyoshi Takagi, editors, PQCrypto 2017, volume 10346 of LNCS, pages 69-89. Springer, 2017.

29. Aggelos Kiayias, Alexander Russell, Bernardo David, and Roman Oliynykov. Ouroboros: A Provably Secure Proof-of-Stake Blockchain Protocol, pages 357-388. Springer International Publishing, Cham, 2017.

30. Hugo Krawczyk. Sigma: The 'sign-and-mac'approach to authenticated diffiehellman and its use in the ike protocols. In Annual International Cryptology Conference, pages 400-425. Springer, 2003.

31. Tanja Lange. Initial recommendations of long-term secure post-quantum systems. PQCRYPTO. EU. Horizon, 2020, 2015.

32. Vadim Lyubashevsky, Chris Peikert, and Oded Regev. On Ideal Lattices and Learning with Errors over Rings, pages 1-23. Springer Berlin Heidelberg, Berlin, Heidelberg, 2010. 
33. Carl Lndahl, Thomas Johansson, Masoumeh Koochak Shooshtari, Mahmoud Ahmadian-Attari, and Mohammad Reza Aref. Squaring attacks on McEliece public-key cryptosystems using quasi-cyclic codes of even dimension. Designs, Codes and Cryptography, 80(2):359-377, 2016.

34. Ingo Von Maurich, Tobias Oder, and Tim Güneysu. Implementing qc-mdpc mceliece encryption. ACM Trans. Embed. Comput. Syst., 14(3):44:1-44:27, April 2015.

35. Alexander May and Ilya Ozerov. On computing nearest neighbors with applications to decoding of binary linear codes. In Elisabeth Oswald and Marc Fischlin, editors, Advances in Cryptology - EUROCRYPT 2015, Part I, volume 9056 of LNCS, pages 203-228. Springer, 2015.

36. R. J. McEliece. A public-key cryptosystem based on algebraic coding theory. Deep Space Network Progress Report, 44:114-116, January 1978.

37. Daniele Micciancio. Improving lattice based cryptosystems using the hermite normal form. Cryptography and lattices, pages 126-145, 2001.

38. V. S. Miller. Use of elliptic curves in cryptography. In Advances in cryptology (CRYPTO 85), pages 417-426, New York, USA, 1986. Springer-Verlag.

39. R. Misoczki and P. S. L. M. Barreto. Compact McEliece keys from Goppa codes. In Selected Areas in Cryptography, pages 376-392, 2009.

40. R. Misoczki, J.-P. Tillich, N. Sendrier, and P. L.S.M. Barreto. MDPC-McEliece: New McEliece variants from moderate density parity-check codes. In IEEE International Symposium on Information Theory - ISIT'2013, pages 2069-2073, Istambul, Turkey, 2013. IEEE.

41. C. Monico, J. Rosenthal, and A. Shokrollahi. Using low density parity check codes in the McEliece cryptosystem. In IEEE International Symposium on Information Theory (ISIT'2000), page 215, Sorrento, Italy, 2000. IEEE.

42. Chris Peikert. Lattice cryptography for the internet. In International Workshop on Post-Quantum Cryptography, pages 197-219. Springer, 2014.

43. Chris Peikert. A decade of lattice cryptography. Foundations and Trends $\mathbb{R}$ in Theoretical Computer Science, 10(4):283-424, 2016.

44. Edoardo Persichetti. Compact mceliece keys based on quasi-dyadic srivastava codes. Journal of Mathematical Cryptology, 6(2):149-169, 2012.

45. Edoardo Persichetti. Secure and anonymous hybrid encryption from coding theory. In Philippe Gaborit, editor, Post-Quantum Cryptography: 5th International Workshop, PQCrypto 2013, Limoges, France, June 4-7, 2013. Proceedings, pages 174-187, Berlin, Heidelberg, 2013. Springer Berlin Heidelberg.

46. E. Prange. The use of information sets in decoding cyclic codes. IRE Transactions, IT-8:S5-S9, 1962.

47. Oded Regev. On lattices, learning with errors, random linear codes, and cryptography. Journal of the ACM (JACM), 56(6):34, 2009.

48. R. L. Rivest, A. Shamir, and L. M. Adleman. A method for obtaining digital signatures and public-key cryptosystems. Communications of the ACM, 21(2):120126, 1978.

49. N. Sendrier. Decoding one out of many. In B.-Y. Yang, editor, PQCrypto 2011, volume 7071 of $L N C S$, pages 51-67. Springer, 2011.

50. Karen Seo and Stephen Kent. Security architecture for the internet protocol. Status: Proposed Standard, 2005.

51. P. W. Shor. Polynomial-time algorithms for prime factorization and discrete logarithms on a quantum computer. SIAM Journal on Computing, 26(5):1484-1509, 1997. 
52. Rodolfo Canto Torres and Nicolas Sendrier. Analysis of information set decoding for a sub-linear error weight. In Tsuyoshi Takagi, editor, PQCrypto 2016, volume 9606 of $L N C S$, pages 144-161. Springer, 2016.

53. Ingo Von Maurich and Tim Güneysu. Lightweight code-based cryptography: QCMDPC McEliece encryption on reconfigurable devices. In Proceedings of the conference on Design, Automation 85 Test in Europe, page 38. European Design and Automation Association, 2014.

54. Ingo Von Maurich and Tim Güneysu. Towards side-channel resistant implementations of QC-MDPC McEliece encryption on constrained devices. PQCrypto, 2014:266-282, 2014. 